Seng-Kee Chua, Department of Mathematics, National University of Singapore, 10, Kent Ridge Crescent, Singapore 0511

\title{
WEIGHTED SOBOLEV INEQUALITIES OF MIXED NORM
}

\begin{abstract}
We obtain weighted Sobolev inequalities of mixed norm on parallelepipeds and then generalize them to product of domains satisfying the chain condition.
\end{abstract}

\section{Introduction}

Recently, there has been quite a number of papers on weighted Sobolev's inequalities. For example, Brown and Hinton [1], [2], Chanillo and Wheeden [4], Sawyer and Wheeden [16] and Chua [5], [6] and [7]. Also, Shi and Torchinsky [17] proved some weighted Sobolev inequalities on parallelepipeds. We would also like to study weighted Sobolev inequalities on product spaces, namely we would like to generalize those inequalities to mixed norm inequalities.

In what follows, $C$ denotes various positive constants. They may differ even in a same string of estimates. Moreover, sometimes, we will use $C(\alpha, \beta, \ldots)$ instead of $C$ to emphasize that the constant is depending on $\alpha, \beta, \ldots$ Cubes are open with sides parallel to the coordinate axes. By a weight, we mean a positive locally integrable function. Following [17], we say that a weight $w$ on $\mathbb{R}^{n} \times \mathbb{R}^{m}$ satisfies Muckenhoupt's $A_{p}\left(\mathbb{R}^{n} \times \mathbb{R}^{m}\right)$ condition, $1<p<\infty$ if

$$
\frac{1}{|I \times J|}\left(\int_{J} \int_{I} w(x, y) d x d y\right)^{1 / p}\left(\int_{J} \int_{I} w^{-1 /(p-1)} d x d y\right)^{(p-1) / p} \leq C
$$

Key Words: Poincaré inequalities and Sobolev's inequalities of mixed norm, doubling and reverse doubling weights on product spaces, $A_{p}$ weights, domains satisfying the Boman chain condition, Lipschitz domains

Mathematical Reviews subject classification: Primary: 26D10, 46E35

Received by the editors March 15, 1995 
for all parallelepipeds $I \times J, I \subset \mathbb{R}^{n}, J \subset \mathbb{R}^{m}$, are cubes. (This definition is actually equivalent to that given in [9] or [10].) Moreover, we say that a weight $w$ is doubling on $\mathbb{R}^{n} \times \mathbb{R}^{m}$ if

$$
w(2 I, 2 J) \leq C w(I, J) \text { for all open cubes } I \subset \mathbb{R}^{n}, J \subset \mathbb{R}^{m}
$$

where $w(I, J)=\int_{I} \int_{J} w(x, y) d x d y$. Also, we say that a weight $w$ is reverse doubling on $\mathbb{R}^{n} \times \mathbb{R}^{m}$ if there exists an $\varepsilon>0$ such that

$$
w(I, J) \geq C\left(\frac{|I \times J|}{\left|I_{0} \times J_{0}\right|}\right)^{\varepsilon} w\left(I_{0}, J_{0}\right)
$$

for all cubes $I, I_{0}, J, J_{0}, I \subseteq I_{0} \subset \mathbb{R}^{n}, J \subseteq J_{0} \subset \mathbb{R}^{m}$. Note that $A_{p}$ weights are doubling and hence reverse doubling on $\mathbb{R}^{n} \times \mathbb{R}^{m}$. In what follows, $Q$, $I, J$ are always cubes and $p^{\prime}$ will denote $p /(p-1)$ if $1<p<\infty$. Finally, if $f$ is a function defined on an open set of $\mathbb{R}^{n} \times \mathbb{R}^{m}$ such that it is weakly differentiable, we denote by $\nabla_{1} f(x, y)$ the partial gradient of $f$ containing the $x$-derivatives; similarly for $\nabla_{2} f(x, y)$, the partial gradient of $f$ containing the $y$-derivatives.

Let $1 \leq q_{1}, q_{2}<\infty$ and let $w$ be a weight on $\mathbb{R}^{n} \times \mathbb{R}^{m}$. If $E$ is a measurable set in $\mathbb{R}^{n} \times \mathbb{R}^{m}$, we will let

$$
\|f\|_{L_{w}^{\tilde{q}(E)}}=\left(\int_{\mathbb{R}^{n}}\left[\int_{\mathbb{R}^{m}}|f(x, y)|^{q_{1}} \chi_{E}(x, y) w(x, y) d y\right]^{q_{2} / q_{1}} d x\right)^{1 / q_{2}}
$$

and

$$
\|f\|_{L_{w}^{\hat{q}(E)}}=\left(\int_{\mathbb{R}^{m}}\left[\int_{\mathbb{R}^{n}}|f(x, y)|^{q_{2}} \chi_{E}(x, y) w(x, y) d x\right]^{q_{1} / q_{2}} d y\right)^{1 / q_{1}} .
$$

Moreover, we will define $\|f\|_{L_{v}^{\hat{p}(E)}}$ and $\|f\|_{L_{\mu}^{\hat{r}(E)}}$ similarly.

Shi and Torchinsky proved the following.

Theorem 1.1 [17, Theorem 1] Let $1<p \leq q<\infty$. Assume $f$ is a Lipschitz continuous function on a parallelepiped $R=I \times J$ and suppose that the doubling weights $w, v$ satisfy the following conditions:

(i) $v \in A_{p}\left(\mathbb{R}^{n} \times \mathbb{R}^{m}\right)$ and

(ii) $\left(\frac{I^{\prime} \mid}{|\tilde{I}|}\right)^{\alpha / n}\left(\frac{\left|J^{\prime}\right|}{|\tilde{J}|}\right)^{\beta / m}\left(\frac{w\left(I^{\prime}, J^{\prime}\right)}{w(\tilde{I}, \tilde{J})}\right)^{1 / q} \leq C\left(\frac{v\left(I^{\prime}, J^{\prime}\right)}{v(\tilde{I}, \tilde{J})}\right)^{1 / p}$ for all cubes $I^{\prime} \subset \tilde{I} \subset$ $\mathbb{R}^{n}, J^{\prime} \subset \tilde{J} \subset \mathbb{R}^{m}$, with $0<\alpha, \beta<1$, and $\alpha+\beta<1$. 
If $f_{R}$ denotes the average of $f$ over $R$, then

$$
\left.\frac{1}{w(R)^{1 / q}}\left\|f-\left.f_{R}\right|_{L_{w}^{q}(R)} \leq C \frac{|I|^{1 / n}}{v(R)^{1 / p}}\right\| \nabla_{1} f\right|_{L_{v}^{p}(R)}+C \frac{|J|^{1 / m}}{v(R)^{1 / p}}\left\|\nabla_{2} f\right\|_{L_{v}^{p}(R)} .
$$

We will first generalize this to mixed norm inequalities on parallelepipeds (see Theorem 1.2) and then to inequalities on product of domains satisfying the chain condition (see Theorem 3.9). Finally, in the case $u=v=w \in$ $A_{p}\left(\mathbb{R}^{n} \times \mathbb{R}^{m}\right)$, we obtain a simple weighted Sobolev inequality on product of domains satisfying the chain condition (see Corollary 3.12 and Definition 3.6).

Theorem 1.2 Let $1<r_{1}, r_{2}, p_{1}, p_{2} \leq q_{1}, q_{2}<\infty$, and $p_{i}, r_{i}<q_{i}$ for $i=1,2$. Let $\mu, v, w$ be weights such that $\mu^{\prime}, v^{\prime}$, $\tilde{w}$ are reverse doubling on $\mathbb{R}^{n} \times \mathbb{R}^{m}$ where

$$
\begin{aligned}
\mu^{\prime}(I, J) & =\int_{J}\left[\int_{I} \mu^{-\frac{1}{r_{2}-1}}(x, y) d x\right]^{r_{1}^{\prime} / r_{2}^{\prime}} d y \\
v^{\prime}(I, J) & =\int_{J}\left[\int_{I} v^{-\frac{1}{p_{2}-1}}(x, y) d x\right]^{p_{1}^{\prime} / p_{2}^{\prime}} d y \\
\tilde{w}(I, J) & =\int_{I}\left[\int_{J} w(x, y) d y\right]^{q_{2} / q_{1}} d x .
\end{aligned}
$$

Suppose further that there exist $0<\alpha, \beta, \gamma, \delta<1$ with $\alpha+\beta \leq 1$, and $\gamma+\delta \leq 1$ such that

$$
\begin{aligned}
& \left(\frac{\tilde{w}\left(I_{0}, J^{\prime}\right)}{\tilde{w}\left(I_{0}, J\right)}\right)^{1 / q_{2}}\left(\frac{\left|J^{\prime}\right|}{|J|}\right)^{\frac{\beta}{m}-1}\left(\frac{v^{\prime}\left(I, J^{\prime}\right)}{v^{\prime}(I, J)}\right)^{1 / p_{1}^{\prime}} \leq C \\
& \left(\frac{\tilde{w}\left(I^{\prime}, J\right)}{\tilde{w}(I, J)}\right)^{1 / q_{2}}\left(\frac{\left|I^{\prime}\right|}{|I|}\right)^{\frac{\alpha}{n}-1}\left(\frac{v^{\prime}\left(I^{\prime}, \tilde{J}\right)}{v^{\prime}(I, \tilde{J})}\right)^{1 / p_{1}^{\prime}} \leq C \\
& \left(\frac{\tilde{w}\left(\tilde{I}, J^{\prime}\right)}{\tilde{w}(\tilde{I}, J)}\right)^{1 / q_{2}}\left(\frac{\left|J^{\prime}\right|}{|J|}\right)^{\frac{\delta}{m}-1}\left(\frac{\mu^{\prime}\left(I, J^{\prime}\right)}{\mu^{\prime}(I, J)}\right)^{1 / r_{1}^{\prime}} \leq C \\
& \left(\frac{\tilde{w}\left(I^{\prime}, J\right)}{\tilde{w}(I, J)}\right)^{1 / q_{2}}\left(\frac{\left|I^{\prime}\right|}{|I|}\right)^{\frac{\gamma}{n}-1}\left(\frac{\mu^{\prime}\left(I^{\prime}, \tilde{J}\right)}{\mu^{\prime}(I, \tilde{J})}\right)^{1 / r_{1}^{\prime}} \leq C
\end{aligned}
$$

for all cubes $I, I^{\prime}, \tilde{I}$ in $\mathbb{R}^{n}, J, J^{\prime}, \tilde{J}$ in $\mathbb{R}^{m}$, such that $I^{\prime} \subset I$ and $J^{\prime} \subset J$. 
Then

$$
\begin{aligned}
& \frac{|I||J|}{\tilde{w}(I, J)^{1 / q_{2}}}\left\|f-f_{I \times J}\right\|_{L_{w}^{\tilde{q}}(I \times J)} \\
& \quad \leq C v^{\prime}(I, J)^{1 / p_{1}^{\prime}}|I|^{1 / n}\left\|\left.\nabla_{1} f\right|_{L_{v}^{\hat{p}}(I \times J)}+\mu^{\prime}(I, J)^{1 / r_{1}^{\prime}}|J|^{1 / m}|| \nabla_{2} f\right\|_{L_{\mu}^{\hat{p}}(I \times J)}
\end{aligned}
$$

for all parallelepipeds $I \times J$ in $\mathbb{R}^{n} \times \mathbb{R}^{m}$ and any Lipschitz continuous function $f$ on $I \times J$.

\section{Remarks 1.3}

(a) When $1<p<q$, we are able to extend Theorem 1.1 by including the case $\alpha+\beta=1$ under slightly stronger condition. See Theorem 3.10.

(b) We can also obtain similar inequality if we take $f_{I \times J}$ to be the weighted average

$$
a_{I \times J}=\frac{1}{w(I, J)} \int_{I} \int_{J} f(x, y) w(x, y) d y d x .
$$

Indeed, by the triangle inequality and Hölder's inequality, we can obtain

$$
\begin{aligned}
\| f- & a_{I \times J}\left\|_{L_{w}^{\tilde{q}(I \times J)}} \leq\right\| f-f_{I \times J} \|_{L_{w}^{\tilde{q}}(I \times J)} \\
& \times\left[1+\tilde{w}(I, J)^{1 / q_{2}}\left(\int_{I}\left[\int_{J} w(x, y) d y\right]^{q_{2}^{\prime} / q_{1}^{\prime}} d x\right)^{1 / q_{2}^{\prime}} w(I, J)^{-1}\right] .
\end{aligned}
$$

\section{Some Preliminary Results}

First, the following theorem is a consequence of the proof of Theorem 1B in [16].

Theorem 2.1 Let $1<p<q<\infty, 0<\alpha<n$ and let $v$ and $w$ be weights such that $w$ and $v^{-1 /(p-1)}$ are reverse doubling on $\mathbb{R}^{n}$. Suppose further that there exists an $A>0$ such that

$$
w(Q)^{1 / q}|Q|^{\frac{\alpha}{n}-1}\left(v^{-1 /(p-1)}(Q)\right)^{1 / p^{\prime}} \leq A \text { for all cubes } Q \text { in } \mathbb{R}^{n} .
$$

Then there exists a positive constant $C_{0}$ depending only on the reverse doubling constants and $A$ such that

$$
\left\|I_{\alpha} f\right\|_{L_{w}^{q}\left(\mathbb{R}^{n}\right)} \leq C_{0}\|f\|_{L_{v}^{p}\left(\mathbb{R}^{n}\right)}
$$

for all $f \in L_{v}^{p}\left(\mathbb{R}^{n}\right)$ where $I_{\alpha} f(x)=\int_{\mathbb{R}^{n}} \frac{|f(y)|}{|x-y|^{n-\alpha}} d y$. 
Proof. Theorem 1B of [16] states that the constant $C_{0}$ in (2.1) depends only on $v$ and $w$. However, the proof given in [16] actually proves that $C_{0}$ depends only on the reverse doubling constants and $A$.

The previous theorem immediately implies the following.

Corollary 2.2 Let $1<p<q<\infty, 0<\alpha<n$ and let $v$ and $w$ be weights such that $w$ and $v^{-1 /(p-1)}$ are reverse doubling on $\mathbb{R}^{n}$. Suppose further that there exists an $A>0$ such that

$$
\left(\frac{w\left(Q^{\prime}\right)}{w(Q)}\right)^{1 / q}\left(\frac{\left|Q^{\prime}\right|}{|Q|}\right)^{\frac{\alpha}{n}-1}\left(\frac{v^{-1 /(p-1)}\left(Q^{\prime}\right)}{v^{-1 /(p-1)}(Q)}\right)^{1 / p^{\prime}} \leq A
$$

for all cubes $Q, Q^{\prime}$ in $\mathbb{R}^{n}, Q^{\prime} \subset Q$. Then there exists a $C>0$ depending only on the reverse doubling constants and $A$ such that

$$
\left\|I_{\alpha} f\right\|_{L_{w}^{q}(Q)} \leq C w(Q)^{1 / q}|Q|^{\frac{\alpha}{n}-1}\left(v^{-1 /(p-1)}(Q)\right)^{1 / p^{\prime}}\|f\|_{L_{v}^{p}(Q)}
$$

for all $f \in L_{v}^{p}\left(\mathbb{R}^{n}\right)$ and all cubes $Q$ in $\mathbb{R}^{n}$.

Next, let us state a lemma from [17].

Lemma 2.3 [17, Corollary 1] Let $0<\gamma, \lambda<1$ and $f$ is a Lipschitz continuous function in $R=I \times J$ and $(x, y) \in R$. Then there exists a constant $C>0$ independent of $f$ and $R$ such that

$$
\begin{aligned}
\left|f(x, y)-f_{R}\right| \leq & C\left(\frac{|I|^{1 / n}}{|J|^{1 / m}}\right)^{\gamma} \iint_{R} \frac{\left|\nabla_{1} f(u, z)\right|}{|u-x|^{n-(1-\gamma)}|z-y|^{m-\gamma}} d u d z \\
& +C\left(\frac{|J|^{1 / m}}{|I|^{1 / n}}\right)^{\lambda} \iint_{R} \frac{\left|\nabla_{2} f(u, z)\right|}{|u-x|^{n-\lambda}|z-y|^{m-(1-\lambda)}} d u d z .
\end{aligned}
$$

Finally, let us prove a mixed norm inequality for fractional integrals on product spaces.

Lemma 2.4 Let $0<\alpha<n, 0<\beta<m, 1<p_{1}, p_{2} \leq q_{1}, q_{2}<\infty$ and $p_{i}<q_{i}$ for $i=1,2$. Let $v, w$ be weights such that $v^{\prime}$ and $\tilde{w}$ are reverse doubling on $\mathbb{R}^{n} \times \mathbb{R}^{m}$ where $v^{\prime}$ and $\tilde{w}$ are defined as in Theorem 1.2. Suppose further that they satisfy (iii) and (iv) in Theorem 1.2. Then there exists $C>0$ such that for any parallelepiped $I \times J$ in $\mathbb{R}^{n} \times \mathbb{R}^{m}$, we have

$$
\begin{aligned}
& \left(\int_{I}\left[\int_{J}\left(\int_{I} \int_{J} \frac{|f(u, z)|}{|u-x|^{n-\alpha}|z-y|^{m-\beta}} d z d u\right)^{q_{1}} w(x, y) d y\right]^{q_{2} / q_{1}} d x\right)^{1 / q_{2}} \\
& \quad \leq C v^{\prime}(I, J)^{1 / p_{1}^{\prime}} \tilde{w}(I, J)^{1 / q_{2}}|I|^{\frac{\alpha}{n}-1}|J|^{\frac{\beta}{m}-1}|| f \|_{L_{v}^{\hat{p}}(I \times J)} .
\end{aligned}
$$


Proof. Let $I \times J$ be a parallelepiped in $\mathbb{R}^{n} \times \mathbb{R}^{m}$. Let

$$
\begin{aligned}
v_{0}(y) & =\left(v^{\frac{-1}{p_{2}-1}}(I, y)\right)^{-p_{1} / p_{2}^{\prime}} \text { and let } \\
k(x) & =w(x, J)^{1 / q_{1}}|J|^{\frac{\beta}{m}-1}\left(v_{0}^{\frac{-1}{p_{1}-1}}(J)\right)^{1 / p_{1}^{\prime}}=w(x, J)^{1 / q_{1}}|J|^{\frac{\beta}{m}-1}\left(v^{\prime}(I, J)\right)^{1 / p_{1}^{\prime}} .
\end{aligned}
$$

Note that

$$
\int_{I^{\prime}} k(x)^{q_{2}} d x=\tilde{w}\left(I^{\prime}, J\right)|J|^{\left(\frac{\beta}{m}-1\right) q_{2}}\left(v^{\prime}(I, J)\right)^{q_{2} / p_{1}^{\prime}} .
$$

Thus,

$$
\begin{aligned}
& \left(\int_{I}\left[\int_{J}\left(\int_{J} \int_{I} \frac{|f(u, z)|}{|u-x|^{n-\alpha}|z-y|^{m-\beta}} d u d z\right)^{q_{1}} w(x, y) d y\right]^{q_{2} / q_{1}} d x\right)^{1 / q_{2}} \\
& \leq C\left(\int_{I}\left[\int_{J}\left(\int_{I} \frac{|f(u, y)|}{|u-x|^{n-\alpha}} d u\right)^{p_{1}} v_{0}(y) d y\right]^{q_{2} / p_{1}} k(x)^{q_{2}} d x\right)^{\frac{p_{1}}{q_{2} \frac{1}{p_{1}}}}
\end{aligned}
$$

by (iii) and Corollary 2.2

$\leq C\left(\int_{J}\left[\int_{I}\left(\int_{I} \frac{|f(u, y)|}{|u-x|^{n-\alpha}} d u\right)^{q_{2}} k(x)^{q_{2}} d x\right]^{p_{1} / q_{2}} v_{0}(y) d y\right)^{1 / p_{1}}$

by Minkowski's inequality since $q_{2} \geq p_{1}$

$$
\begin{aligned}
\leq & C\left(\int_{J}\left[\int_{I}|f|^{p_{2}} v(x, y) d x\right]^{p_{1} / p_{2}}\left[\int_{I} v^{\frac{-1}{p_{2}-1}}(x, y) d x\right]^{p_{1} / p_{2}^{\prime}} v_{0}(y) d y\right)^{1 / p_{1}} \\
& \times \tilde{w}(I, J)^{1 / q_{2}}|J|^{\frac{\beta}{m}-1}|I|^{\frac{\alpha}{n}-1} v^{\prime}(I, J)^{1 / p_{1}^{\prime}} \text { by (iv) and Corollary } 2.2
\end{aligned}
$$

$\leq C|I|^{\frac{\alpha}{n}-1}|J|^{\frac{\beta}{m}-1} \tilde{w}(I, J)^{1 / q_{2}} v^{\prime}(I, J)^{1 / p_{1}^{\prime}}\left(\int_{J}\left[\int_{I}|f|^{p_{2}} v(x, y) d x\right]^{p_{1} / p_{2}} d y\right)^{1 / p_{1}}$.

We can now prove Theorem 1.2. 
Proof of Theorem 1.2. First note that by Lemma 2.4,

$$
\begin{aligned}
& \left(\frac{|I|^{1 / n}}{|J|^{1 / m}}\right)^{\alpha} \\
& \times\left(\int_{I}\left[\int_{J}\left(\int_{I} \int_{J} \frac{\left|\nabla_{1} f(u, z)\right|}{|u-x|^{n-(1-\alpha)}|z-y|^{m-\alpha}} d z d u\right)^{q_{1}} w(x, y) d y\right]^{q_{2} / q_{1}} d x\right)^{1 / q_{2}} \\
& \leq C\left(|I|^{1 / n} /|J|^{1 / m}\right)^{\alpha}|| \nabla_{1} f \|_{\hat{p}_{v}(I \times J)} v^{\prime}(I, J)^{1 / p_{1}^{\prime}}|I|^{\frac{1-\alpha}{n}-1}|J|^{\frac{\alpha}{m}-1} \tilde{w}(I, J)^{1 / q_{2}} \\
& =C|I|^{\frac{1}{n}-1}|J|^{-1} \tilde{w}(I, J)^{1 / q_{2}} v^{\prime}(I, J)^{1 / p_{1}^{\prime}|| \nabla_{1} f \|_{L_{v}^{\hat{p}}(I \times J)} .}
\end{aligned}
$$

Theorem 1.2 now follows from Lemma 2.3 as estimates for $\nabla_{2} f$ can be obtained similarly.

\section{Sobolev Inequalities on Product of Certain Domains}

Since one of our main tools will be projection of functions into polynomials, we will first state some theorems regarding polynomials. The following lemma is a generalization of [18, Chapter 3, Lemma 7].

Lemma 3.1 If $\mu$ is a doubling measure on $\mathbb{R}^{n} \times \mathbb{R}^{m}$ and $k$ is a positive integer, then there exists a positive constant $s_{0}(n, m, \mu)$ such that if $0<s<s_{0}$, then for any parallelepiped $I \times J$ in $\mathbb{R}^{n} \times \mathbb{R}^{m}$ and $\lambda>0$ such that

$$
\mu(\{(x, y) \in I \times J:|p(x, y)|>\lambda\}) \leq s \mu(I, J)
$$

we have $\sup _{(x, y) \in I \times J}|p(x, y)| \leq C \lambda$, where $p$ is any polynomial of degree $\leq k$ and $C$ is a constant independent of $\lambda, I, J$ and $p$.

In particular if $\mu=\tilde{w}$, note that

$$
\tilde{w}(\{(x, y) \in I \times J:|p(x, y)|>\lambda\}) \leq\left(\frac{1}{\lambda}\|p\|_{L_{w}^{\tilde{q}}(I \times J)}\right)^{q_{2}} .
$$

Hence, $\|p\|_{L_{(I \times J)}^{\infty}} \leq \frac{C}{\tilde{w}(I, J)^{1 / q_{2}}}\|p\|_{L_{w}^{\tilde{q}}(I \times J)}$ with $C$ depending only on $k$ and the doubling constant of $\tilde{w}$ (and the dimensions $m$ and $n$ ).

Proof of Lemma 3.1. We will follow the idea of the proof of [18, Chapter 3, Lemma 7]. Let $p$ be a polynomial of degree $N, N \leq k$, and let $R=I \times J$ be a parallelepiped in $\mathbb{R}^{n} \times \mathbb{R}^{m}$. First note that it suffices to show that there are 
constants $C_{1}, C_{2}>0$ depending only on the doubling constant of the weight $\mu, k$ and the dimensions $n, m$ such that

$$
\mu\left\{z \in R:|p(z)|>c_{1}\|p\|_{L^{\infty}(R)}\right\} \geq C_{2} \mu(R) .
$$

Let $z_{0} \in R$ such that $\left|p\left(z_{0}\right)\right|=\|p\|_{L^{\infty}(R)}$ and let $R_{0}=I_{0} \times J_{0} \subset R$ such that $z_{0} \in R_{0}, l\left(I_{0}\right)=l(I) / 2, l\left(J_{0}\right)=l(J) / 2$. By a change of coordinates and scaling if necessary we may assume that $z_{0}=0$ and is a corner of $R_{0}$ and $R_{0}=\left\{(x, y) \in \mathbb{R}^{n} \times \mathbb{R}^{m}: 0 \leq x_{i} \leq 1,0 \leq y_{j} \leq \alpha\right.$ for $i=1, \ldots, n$, $j=1, \ldots, m\}$ for some $\alpha>0$. Let

$$
E=\left\{(x, y) \in R_{0}:|(x, y)| \leq \sqrt{n+m \alpha^{2}} /(8 k)^{k},|\alpha x| / 2 \leq|y| \leq 2|\alpha x|\right\} .
$$

To prove (3.1), let us note that it suffices to show that $|p(z)|>\left|p\left(z_{0}\right)\right| / 2^{k}$ for $z \in E$.

Let $z^{\prime}=\left(x^{\prime}, y^{\prime}\right)$ such that $\left|z^{\prime}\right|=1,\left|\alpha x^{\prime}\right| / 2 \leq\left|y^{\prime}\right| \leq 2\left|\alpha x^{\prime}\right|$. We then let $t^{\prime}=\sup \left\{t \in \mathbb{R}: t z^{\prime} \in R_{0}\right\}$ and $g(t)=p\left(t z^{\prime}\right)$ for $t \in \mathbb{R}$. Then $g$ is a polynomial of degree $N \leq k$. Consequently, $g(t)=a\left(t-a_{1}\right) \cdots\left(t-a_{N}\right)$ and

$$
\frac{\left|a\left(t-a_{1}\right) \cdots\left(t-a_{N}\right)\right|}{\left|a a_{1} \cdots a_{N}\right|}=\frac{\left|p\left(t z^{\prime}\right)\right|}{\left|p\left(z_{0}\right)\right|} \leq 1 \quad \text { for } t \in\left[0, t^{\prime}\right] .
$$

Next, since $t^{\prime} \geq \sqrt{n+m \alpha^{2}} / 2$, it is easy to see that there exists $t_{0} \in\left[0, t^{\prime}\right]$ such that

$$
\left|t_{0}-a_{j}\right| \geq \sqrt{n+m \alpha^{2}} / 4 N \geq \sqrt{n+m \alpha^{2}} / 4 k \text { for } j=1,2, \ldots, N .
$$

By considering two cases $\left|a_{j}\right| \geq 2 \sqrt{n+m \alpha^{2}}$ and $\left|a_{j}\right|<2 \sqrt{n+m \alpha^{2}}$, and using the fact that $\left|t_{0}-a_{j}\right| \geq \sqrt{n+m \alpha^{2}} / 4 k$ and $\left|t_{0}\right| \leq \sqrt{n+m \alpha^{2}}$, it is easy to see that $\left|t_{0}-a_{j}\right| /\left|a_{j}\right| \geq 1 / 8 k$ for all $j$. Hence by (3.2), we see that

$$
\frac{\left|t_{0}-a_{j}\right|}{\left|a_{j}\right|} \leq(8 k)^{N-1} \leq(8 k)^{k-1}
$$

for all $j$. Thus

$$
\left|a_{j}\right| \geq \frac{\left|t_{0}-a_{j}\right|}{(8 k)^{k-1}} \geq \frac{\sqrt{n+m \alpha^{2}} / 4 k}{(8 k)^{k-1}}=\frac{2 \sqrt{n+m \alpha^{2}}}{(8 k)^{k}}
$$

for all $j$. Now if $0 \leq t \leq \sqrt{n+m \alpha^{2}} /(8 k)^{k}$, then

$$
\left|a_{j}\right| \leq\left|a_{j}-t\right|+t \leq\left|a_{j}-t\right|+\frac{\left|a_{j}\right|}{2} \text { and hence } \frac{\left|a_{j}-t\right|}{\left|a_{j}\right|} \geq \frac{1}{2}
$$


for all $j$ and therefore $|g(t)| /|g(0)| \geq 1 / 2^{N} \geq 1 / 2^{k}$. Thus $|p(z)| \geq\left|p\left(z_{0}\right)\right| / 2^{k}$ if $z \in E$ and this completes the proof.

Next the following is a generalized form of [5, Theorem 2.2].

Lemma 3.2 Let $R$ be a parallelepiped and let $E$ be a measurable set in $R$ with $|E|>\gamma|R|$. If $p$ is a polynomial of degree $\leq k$, then

$$
\|p\|_{L^{\infty}(E)} \geq C(\gamma, k)\|p\|_{L^{\infty}(R)} .
$$

Proof. In [5], (3.3) was proved to hold for $R=Q_{0}$ the unit cube in $\mathbb{R}^{n} \times \mathbb{R}^{m}$ with center at the origin. Now if $R$ is any parallelepiped, same as the proof in [5], there exists an affine transformation $T$ mapping $R$ isomorphically onto $Q_{0}$. It is now easy to see that (3.3) holds.

The following lemma is an immediate consequence of the previous lemma.

Lemma 3.3 Let $E$ and $F$ be parallelepipeds such that $E \subset F$ and $|E|>\gamma|F|$. If $p$ is a polynomial of degree $\leq k$, then

$$
\|p\|_{L^{\infty}(E)} \geq C(\gamma, k)\|p\|_{L^{\infty}(F)} .
$$

The next theorem is an immediate consequence of Lemmas 3.1 and 3.3.

Theorem 3.4 Let $E$ and $F$ be parallelepipeds such that $E \subset F$ and $|E|>$ $\gamma|F|$. Let $1 \leq q_{1}, q_{2}<\infty$. If $w$ is a weight such that $\tilde{w}$ is doubling on $\mathbb{R}^{n} \times \mathbb{R}^{m}$ with doubling constant $A$, and $p$ is a polynomial of degree $\leq k$, then

$$
\|p\|_{L_{w}^{\tilde{q}(F)}} \leq C(\gamma, m, n, k, A)\left(\frac{\tilde{w}(F)}{\tilde{w}(E)}\right)^{1 / q_{2}}\|p\|_{L_{w}^{\tilde{q}}(E)} .
$$

Lemma 3.5 [6, Lemma 2.5] or [19, Lemma 2.3] Let $w$ be a doubling measure on $\mathbb{R}^{n}$ with doubling constant $A$. Let $\left\{Q_{\alpha}\right\}_{\alpha \in I}$ be an arbitrary family of cubes in $\mathbb{R}^{n}$. If $\left\{a_{\alpha}\right\}_{\alpha \in I}$ is a family of non-negative real numbers, then for $1 \leq p<\infty$ and $N \geq 1$, we have

$$
\left\|\sum_{\alpha} a_{\alpha} \chi_{N Q_{\alpha}}\right\|_{L_{w}^{p}\left(\mathbb{R}^{n}\right)} \leq C(A, n, p, N)\left\|\sum_{\alpha} a_{\alpha} \chi_{Q_{\alpha}}\right\|_{L_{w}^{p}\left(\mathbb{R}^{n}\right)} .
$$

Note that even though it was stated in the references that the constant in (3.4) depends on $w, n, p$ and $N$, however, the constant actually depends only on $n, p, N$ and the doubling constant $A$. 
Definition 3.6 [12] An open set $\Omega$ in $\mathbb{R}^{n}$ is said to be a member of $\mathcal{F}(\sigma, N)$, $\sigma \geq 1, N \geq 1$, if there exists a covering $W$ of $\Omega$ consisting of open cubes such that:

(a) $\sum_{Q \in W} \chi_{\sigma Q}(x) \leq N \chi_{\Omega}(x) \quad \forall x \in \mathbb{R}^{n}$.

(b) There is a 'central cube' $Q_{0} \in W$ that can be connected with every cube $Q \in W$ by a finite chain of cubes $Q_{0}, Q_{1}, \ldots, Q_{k(Q)}=Q$ from $W$ such that $Q \subset N Q_{j}$ for $j=0,1, \ldots, k(Q)$. Moreover, $Q_{j} \cap Q_{j+1}$ contains a cube $\tilde{Q}_{j}$ such that $Q_{j} \cup Q_{j+1} \subset N \tilde{Q}_{j}$.

We say that $\Omega$ satisfies the Boman chain condition if $\Omega \in \mathcal{F}(\sigma, N)$ for some $N, \sigma \geq 1$. There are many types of domains that satisfy the Boman chain condition, for example, balls, cubes and John domains (see [12]). Moreover, it is easy to check that bounded $(\varepsilon, \infty)$ domains (see [13] or [5] for the definition) satisfy the Boman chain condition. Hence so do bounded Lipschitz domains.

First let us state a consequence of the proof of Theorem 1.5 in [5].

Theorem 3.7 Let $\sigma, N \geq 1,1 \leq q<\infty$, and $\mathcal{D} \in \mathcal{F}(\sigma, N), \mathcal{D} \subset \mathbb{R}^{n}$ and let $f$ be a measurable function defined on $\mathcal{D}$. Suppose $w$ is a doubling weight on $\mathbb{R}^{n}$ with doubling constant $A$. Let $W$ be a collection of cubes that satisfies the chain condition and let $P(f, Q)$ be a polynomial of degree $<k$ associated to each cube $Q$ in $W$. Then

$$
\left\|f-P\left(f, Q_{0}\right)\right\|_{L_{w}^{q}(\mathcal{D})}^{q} \leq C \sum_{Q \in W}\|f-P(f, Q)\|_{L_{w}^{q}(Q)}^{q}
$$

where $Q_{0}$ is the 'central cube' in $\mathcal{D}$ and $C$ depends only on $n, q, A, k$ and $N$.

Let us generalize this theorem to products of domains satisfying the chain condition.

Theorem 3.8 Let $\sigma, N \geq 1,1 \leq q_{2} \leq q_{1}<\infty$ and $\mathcal{D}_{1}, \mathcal{D}_{2} \in \mathcal{F}(\sigma, N)$, $\mathcal{D}_{1} \subset \mathbb{R}^{n}, \mathcal{D}_{2} \subset \mathbb{R}^{m}$ and let $f$ be a measurable function defined on $\mathcal{D}=\mathcal{D}_{1} \times \mathcal{D}_{2}$. Also, let $v$ be a weight and let $w$ be a doubling weight on $\mathbb{R}^{n} \times \mathbb{R}^{m}$. Let $W_{1}$ $\left(W_{2}\right)$ be a collection of cubes in $\mathcal{D}_{1}\left(\mathcal{D}_{2}\right)$ that satisfies the chain condition. Let $P(f, R)$ be a polynomial of degree $\leq k$ associated to each parallelepiped $R=I \times J$ with $I \in W_{1}, J \in W_{2}$. Then

$$
\left\|f-P\left(f, I_{0} \times J_{0}\right)\right\|_{L_{w}^{\tilde{q}}(\mathcal{D})}^{q_{2}} \leq C \sum_{I \in W_{1}} \sum_{J \in W_{2}}\|f-P(f, I \times J)\|_{L_{w}^{\tilde{q}}(I \times J)}^{q_{2}}
$$

where $I_{0}$ is the 'central cube' in $\mathcal{D}_{1}$ and $J_{0}$ is the 'central cube' in $\mathcal{D}_{2}$ and $C$ depends only on $m, n, q_{1}, q_{2}, w, k$ and $N$. 
Proof. We will modify the proof of Theorem 1.5 in [6]. Let $J \in W_{2}$. For any $I \in W_{1}$, there exists a chain $I_{0}, I_{1}, \ldots, I_{\theta}=I$ that connects $I$ to $I_{0}$ (see Definition 3.6(b)) and

$$
\begin{aligned}
& \left(\int_{I}\left[\int_{J}\left|P(f, I \times J)-P\left(f, I_{0} \times J\right)\right|^{q_{1}} w(x, y) d y\right]^{q_{2} / q_{1}} d x\right)^{1 / q_{2}} \\
& \leq \sum_{k=1}^{\theta}\left(\int_{I}\left[\int_{J}\left|P\left(f, I_{k} \times J\right)-P\left(f, I_{k-1} \times J\right)\right|^{q_{1}} w(x, y) d y\right]^{q_{2} / q_{1}} d x\right)^{1 / q_{2}} \\
& \leq C \sum_{k=1}^{\theta}\left(\frac{\tilde{w}(I, J)}{\tilde{w}\left(I_{k} \cap I_{k-1}, J\right)}\right)^{1 / q_{2}} \\
& \quad \times\left\|P\left(f, I_{k} \times J\right)-P\left(f, I_{k-1} \times J\right)\right\|_{L_{w}^{\tilde{q}}\left(I_{k} \cap I_{k-1} \times J\right)} \text { by Theorem } 3.4 \\
& \leq C \sum_{k=1}^{\theta}\left(\frac{\tilde{w}(I, J)}{\tilde{w}\left(I_{k} \cap I_{k-1}, J\right)}\right)^{1 / q_{2}} \\
& \quad \times\left(\left\|f-P\left(f, I_{k} \times J\right)\right\|_{L_{w}^{\tilde{q}}\left(I_{k} \cap I_{k-1} \times J\right)}+\left\|f-P\left(f, I_{k-1} \times J\right)\right\|_{L_{w}^{\tilde{q}}\left(I_{k} \cap I_{k-1} \times J\right)}\right) \\
& \leq C \sum_{k=0}^{\theta}\left(\frac{\tilde{w}(I, J)}{\tilde{w}\left(I_{k}, J\right)}\right)^{1 / q_{2}}\left\|f-P\left(f, I_{k} \times J\right)\right\|_{L_{w}^{\tilde{q}}\left(I_{k} \times J\right)} .
\end{aligned}
$$

Hence for any $x \in \mathbb{R}^{n}$, we have,

$$
\begin{aligned}
\| P(f, I \times J) & -P\left(f, I_{0} \times J\right) \|_{L_{w}^{\tilde{q}}(I \times J)} \frac{\chi_{I}(x)}{\tilde{w}(I, J)^{1 / q_{2}}} \\
& \leq C \sum_{L \in W_{1}}\left(\frac{\chi_{N L}(x)}{\tilde{w}(L, J)}\right)^{1 / q_{2}}\|f-P(f, L \times J)\|_{L_{w}^{\tilde{q}}(L \times J)} .
\end{aligned}
$$


And

$$
\begin{aligned}
& \sum_{I \in W_{1}}\left\|P(f, I \times J)-P\left(f, I_{0} \times J\right)\right\|_{L_{w}^{\tilde{q}}(I \times J)}^{q_{2}} \\
= & \sum_{I \in W_{1}} \int_{\mathbb{R}^{n}}\left\|P(f, I \times J)-P\left(f, I_{0} \times J\right)\right\|_{L_{w}^{\tilde{q}}(I \times J)}^{q_{2}} \frac{\chi_{I}(x)}{\tilde{w}(I, J)} \tilde{w}(x, J) d x \\
\leq & C \int_{\mathbb{R}^{n}}\left(\sum_{L \in W_{1}} \frac{\chi_{N L}(x)}{\tilde{w}(L, J)^{1 / q_{2}}}\|f-P(f, L \times J)\|_{L_{w}^{\tilde{q}}(L \times J)}\right)^{q_{2}} \tilde{w}(x, J) d x \\
\leq & C \int_{\mathbb{R}^{n}} \sum_{L \in W_{1}} \frac{1}{\tilde{w}(L, J)}\|f-P(f, L \times J)\|_{L_{w}^{\tilde{q}}(L \times J)}^{q_{2}} \chi_{L}(x) \tilde{w}(x, J) d x
\end{aligned}
$$

by Lemma 3.5

$\leq C \sum_{L \in W_{1}} \frac{1}{\tilde{w}(L, J)}\|f-P(f, L \times J)\|_{L_{w}^{\tilde{q}}(L \times J)}^{q_{2}} \int_{\mathbb{R}^{n}} \chi_{L}(x) \tilde{w}(x, J) d x$ by the chain condition (a)

$\leq C \sum_{L \in W_{1}}\|f-P(f, L \times J)\|_{L_{w}^{\tilde{q}}(L \times J)}^{q_{2}}$.

Hence,

$$
\begin{aligned}
& \left\|f-P\left(f, I_{0} \times J\right)\right\|_{L_{w}^{\tilde{q}}\left(\mathcal{D}_{1} \times J\right)}^{q_{2}} \\
\leq & C \sum_{I \in W_{1}}\left(\|f-P(f, I \times J)\|_{L_{w}^{q}(I \times J)}^{q_{2}}+\left\|P(f, I \times J)-P\left(f, I_{0} \times J\right)\right\|_{L_{w}^{\tilde{q}}(I \times J)}^{q_{2}}\right) \\
\leq & C \sum_{L \in W_{1}}\|f-P(f, L \times J)\|_{L_{w}^{\tilde{q}(L \times J)}}^{q_{2}} .
\end{aligned}
$$


Next, by Theorem 3.7,

$$
\begin{aligned}
& \int_{\mathcal{D}_{1}}\left[\int_{\mathcal{D}_{2}}\left|f-P\left(f, I_{0} \times J_{0}\right)\right|^{q_{1}} w(x, y) d y\right]^{q_{2} / q_{1}} d x \\
& \quad \leq C \int_{\mathcal{D}_{1}}\left[\sum_{J \in W_{2}} \int_{J}\left|f-P\left(f, I_{0} \times J\right)\right|^{q_{1}} w(x, y) d y\right]^{q_{2} / q_{1}} d x
\end{aligned}
$$

since there exists an $A>0$ such that $w(x, 2 Q) \leq A w(x, Q)$

for all cubes $Q$ in $\mathbb{R}^{m}$ and almost all $x \in \mathbb{R}^{n}$

$$
\begin{aligned}
& \leq C \sum_{J \in W_{2}} \int_{\mathcal{D}_{1}}\left[\int_{J}\left|f-P\left(f, I_{0} \times J\right)\right|^{q_{1}} w(x, y) d y\right]^{q_{2} / q_{1}} d x \text { since } q_{2} \leq q_{1} \\
& \leq C \sum_{I \in W_{1}} \sum_{J \in W_{2}}\|f-P(f, I \times J)\|_{L_{w}^{\tilde{q}}(I \times J)}^{q_{2}}
\end{aligned}
$$

by the previous estimate.

We can now extend Sobolev's inequalities of mixed norm to products of domains satisfying the chain condition.

Theorem 3.9 Let $\sigma, N \geq 1$ and $\mathcal{D}_{1}, \mathcal{D}_{2} \in \mathcal{F}(\sigma, N)$, and let $f$ be a weakly differentiable functions on $\mathcal{D}=\mathcal{D}_{1} \times \mathcal{D}_{2} \subset \mathbb{R}^{n} \times \mathbb{R}^{m}$. Let $W_{1}\left(W_{2}\right)$ be a collection of cubes in $\mathcal{D}_{1}\left(\mathcal{D}_{2}\right)$ that satisfies the chain condition. Under the assumption of Theorem 1.2 and suppose in addition that $p_{2} \leq p_{1} \leq q_{2} \leq q_{1}$, $r_{2} \leq r_{1} \leq q_{2} \leq q_{1}$, and $\tilde{w}$ is doubling on $\mathbb{R}^{n} \times \mathbb{R}^{m}$ such that

$$
\begin{aligned}
& \left(\frac{\tilde{w}\left(I^{\prime}, J^{\prime}\right)}{\tilde{w}(I, J)}\right)^{1 / q_{2}}\left(\frac{\left|J^{\prime}\right|}{|J|}\right)^{-1}\left(\frac{\left|I^{\prime}\right|}{|I|}\right)^{\frac{1}{n}-1}\left(\frac{v^{\prime}\left(I^{\prime}, J^{\prime}\right)}{v^{\prime}(I, J)}\right)^{1 / p_{1}^{\prime}} \leq A \\
& \left(\frac{\tilde{w}\left(I^{\prime}, J^{\prime}\right)}{\tilde{w}(I, J)}\right)^{1 / q_{2}}\left(\frac{\left|I^{\prime}\right|}{|I|}\right)^{-1}\left(\frac{\left|J^{\prime}\right|}{|J|}\right)^{\frac{1}{m}-1}\left(\frac{\mu^{\prime}\left(I^{\prime}, J^{\prime}\right)}{\mu^{\prime}(I, J)}\right)^{1 / r_{1}^{\prime}} \leq A
\end{aligned}
$$

for all cubes $I, I^{\prime} \in W_{1}, I^{\prime} \subset I$ and all cubes $J, J^{\prime} \in W_{2}, J^{\prime} \subset J$, then

$$
\begin{aligned}
\frac{1}{\tilde{w}\left(I_{0}, J_{0}\right)^{1 / q_{2}}} & \left\|f-f_{I_{0} \times J_{0}}\right\|_{L_{w}^{\tilde{w}}(\mathcal{D})} \\
\leq & C A\left|J_{0}\right|^{-1}\left|I_{0}\right|^{-1}\left(\left.v^{\prime}\left(I_{0}, J_{0}\right)^{1 / p_{1}^{\prime}}\left|I_{0}\right|^{1 / n}|| \nabla_{1} f\right|_{L_{v}^{\hat{p}(\mathcal{D})}}\right. \\
& \left.+\left.\mu^{\prime}\left(I_{0}, J_{0}\right)^{1 / r_{1}^{\prime}}\left|J_{0}\right|^{1 / m}|| \nabla_{2} f\right|_{L_{\mu}^{\hat{r}}(\mathcal{D})}\right)
\end{aligned}
$$

where $I_{0}$ and $J_{0}$ are the 'central cubes' in $W_{1}$ and $W_{2}$ respectively. 
Proof. For any parallelepiped $I \times J$, let

$$
\begin{aligned}
& A_{1}(I, J)=\tilde{w}(I, J)^{1 / q_{2}} v^{\prime}(I, J)^{1 / p_{1}^{\prime}}|I|^{\frac{1}{n}-1}|J|^{-1}, \\
& A_{2}(I, J)=\tilde{w}(I, J)^{1 / q_{2}} \mu^{\prime}(I, J)^{1 / r_{1}^{\prime}}|I|^{-1}|J|^{\frac{1}{m}-1} .
\end{aligned}
$$

By Theorems 3.8 and 1.2, we have

$$
\begin{aligned}
& \left(\int_{\mathcal{D}_{1}}\left(\int_{D_{2}}\left|f(x, y)-f_{I_{0} \times J_{0}}\right|^{q_{1}} w(x, y) d y\right)^{q_{2} / q_{1}} d x\right)^{1 / q_{2}} \\
& \leq\left(\sum_{I \in W_{1}} \sum_{J \in W_{2}} \int_{I}\left[\int_{J}\left|f-f_{I \times J}\right|^{q_{1}} w(x, y) d y\right]^{q_{2} / q_{1}} d x\right)^{1 / q_{2}} \text { since } q_{1} \geq q_{2} \\
& \leq C\left(\sum_{I \in W_{1}} \sum_{J \in W_{2}}\left[A_{1}(I, J)\left\|\nabla_{1} f\right\|_{L_{v}^{\hat{p}}(I \times J)}\right]^{q_{2}}\right)^{\frac{1}{q_{2}}} \\
& +C\left(\sum_{I \in W_{1}} \sum_{J \in W_{2}}\left[A_{2}(I, J)\left\|\nabla_{2} f\right\|_{L_{\mu}^{\hat{r}}(I \times J)}\right]^{q_{2}}\right)^{\frac{1}{q_{2}}}=I+I I . \\
& I \leq C\left(\sum_{I \in W_{1}} \sum_{J \in W_{2}} A_{1}(I, J)^{p_{1}} \int_{J}\left(\int_{I}\left|\nabla_{1} f(x, y)\right|^{p_{2}} v(x, y) d x\right)^{p_{1} / p_{2}} d y\right)^{1 / p_{1}} \\
& \text { since } q_{2} \geq p_{1} \\
& \leq C A_{1}\left(I_{0}, J_{0}\right)\left(\sum_{I \in W_{1}} \sum_{J \in W_{2}} \int_{J}\left(\int_{I}\left|\nabla_{1} f(x, y)\right|^{p_{2}} v(x, y) d x\right)^{p_{1} / p_{2}} d y\right)^{1 / p_{1}}
\end{aligned}
$$

by (3.7) and the chain condition (b)

$$
\leq C A_{1}\left(I_{0}, J_{0}\right)\left(\sum_{J \in W_{2}} \int_{J}\left(\sum_{I \in W_{1}} \int_{I}\left|\nabla_{1} f(x, y)\right|^{p_{2}} v(x, y) d x\right)^{p_{1} / p_{2}} d y\right)^{1 / p_{1}}
$$

since $p_{1} \geq p_{2}$

$$
\leq C A_{1}\left(I_{0}, J_{0}\right)\left(\int_{\mathcal{D}_{2}}\left(\int_{\mathcal{D}_{1}}\left|\nabla_{1} f(x, y)\right|^{p_{2}} v(x, y) d x\right)^{p_{1} / p_{2}} d y\right)^{1 / p_{1}}
$$

by the chain condition (a). Similarly, we obtain

$$
I I \leq C A_{2}\left(I_{0}, J_{0}\right)\left(\int_{\mathcal{D}_{2}}\left(\int_{\mathcal{D}_{1}}\left|\nabla_{2} f(x, y)\right|^{r_{2}} \mu(x, y) d x\right)^{r_{1} / r_{2}} d y\right)^{1 / r_{1}} .
$$


This complete the proof of Theorem 3.9.

\section{Remarks}

(a) In particular, if $1<p<q, v \in A_{p}\left(\mathbb{R}^{n} \times \mathbb{R}^{m}\right)$, we are able to obtain the same conclusion for the case $\alpha+\beta=1$ that is not covered by Theorem 1.1, i.e., [17, Theorem 1] under slightly stronger assumption.

Theorem 3.10 Let $1<p<q<\infty$. Suppose that $v \in A_{p}\left(\mathbb{R}^{n} \times \mathbb{R}^{m}\right)$ and $w$ is a reverse doubling weight on $\mathbb{R}^{n} \times \mathbb{R}^{m}$. Suppose further that there exist $0<\alpha$, $\beta<1$ with $\alpha+\beta \leq 1$ such that

$$
\begin{aligned}
& \left(\frac{w\left(\tilde{I}, J^{\prime}\right)}{w(\tilde{I}, J)}\right)^{1 / q}\left(\frac{\left|J^{\prime}\right|}{|J|}\right)^{\frac{\beta}{m}} \leq C\left(\frac{v\left(I, J^{\prime}\right)}{v(I, J)}\right)^{1 / p} \\
& \left(\frac{w\left(I^{\prime}, J\right)}{w(I, J)}\right)^{1 / q}\left(\frac{\left|I^{\prime}\right|}{|I|}\right)^{\frac{\alpha}{n}} \leq C\left(\frac{v\left(I^{\prime}, \tilde{J}\right)}{v(I, \tilde{J})}\right)^{1 / p}
\end{aligned}
$$

for all cubes $I^{\prime}, \tilde{I}, I$ in $\mathbb{R}^{n}, I^{\prime} \subset I$, and cubes $J^{\prime}, \tilde{J}, J$ in $\mathbb{R}^{m}, J^{\prime} \subset J$. Then

$$
\begin{aligned}
\| f- & f_{I \times J} \|_{L_{w}^{q}(I \times J)} \leq C w(I, J)^{1 / q} v(I, J)^{-1 / p} \\
& \times\left[|I|^{\frac{1}{n}}\left\|\nabla_{1} f\right\|_{L_{v}^{p}(I \times J)}+|J|^{\frac{1}{m}}\left\|\nabla_{2} f\right\|_{L_{v}^{p}(I \times J)}\right]
\end{aligned}
$$

for all parallelepipeds $I \times J$ in $\mathbb{R}^{n} \times \mathbb{R}^{m}$.

Proof. By the $A_{p}$ condition and Hölder's inequality, (vii) and (viii) imply (iii) and (iv). Hence (3.9) follows from Theorem 1.2.

(b) The following corollary now follows from Theorems 1.1, 3.8 and 3.10.

Corollary 3.11 Let $\sigma, N \geq 1$ and $\mathcal{D}_{1}, \mathcal{D}_{2} \in \mathcal{F}(\sigma, N)$, and let $f$ be a weakly differentiable function on $\mathcal{D}=\mathcal{D}_{1} \times \mathcal{D}_{2} \subset \mathbb{R}^{n} \times \mathbb{R}^{m}$. Let $W_{1}\left(W_{2}\right)$ be a collection of cubes in $\mathcal{D}_{1}\left(\mathcal{D}_{2}\right)$ that satisfies the chain condition. Let $1<p \leq q<\infty$. Suppose that $v \in A_{p}\left(\mathbb{R}^{n} \times \mathbb{R}^{m}\right)$ and $w$ is a doubling weight on $\mathbb{R}^{n} \times \mathbb{R}^{m}$. Suppose further that there exist $0<\alpha, \beta<1$ with $\alpha+\beta<1$ such that (i) and (ii) in Theorem 1.1 hold for all cubes $I^{\prime} \subset \tilde{I}$ in $\mathbb{R}^{n}$, and cubes $J^{\prime} \subset \tilde{J}$ in $\mathbb{R}^{m}$. If

$$
\left(\frac{w\left(I^{\prime}, J^{\prime}\right)}{w(I, J)}\right)^{1 / q}\left[\left(\frac{\left|I^{\prime}\right|}{|I|}\right)^{\frac{1}{n}}+\left(\frac{\left|J^{\prime}\right|}{|J|}\right)^{\frac{1}{m}}\right] \leq C\left(\frac{v\left(I^{\prime}, J^{\prime}\right)}{v(I, J)}\right)^{1 / p}
$$

for all cubes $I, I^{\prime} \in W_{1}, I^{\prime} \subset I$ and all cubes $J, J^{\prime} \in W_{2}, J^{\prime} \subset J$, then

$$
\begin{aligned}
& \left\|f-f_{I_{0} \times J_{0}}\right\|_{L_{v}^{p}(\mathcal{D})} \\
& \quad \leq C w(I, J)^{1 / q} v(I, J)^{-1 / p}\left[|I|^{\frac{1}{n}}\left\|\nabla_{1} f\right\|_{L_{v}^{p}(D)}+|J|^{\frac{1}{m}}\left\|\nabla_{2} f\right\|_{L_{v}^{p}(\mathcal{D})}\right]
\end{aligned}
$$


where $I_{0}$ and $J_{0}$ are the 'central cubes' in $W_{1}$ and $W_{2}$ respectively. Moreover, if in addition that $1<p<q<\infty$ and (vii) and (viii) hold for some $0<\alpha$, $\beta<1$ such that $\alpha+\beta \leq 1$, then the above inequality also holds.

Finally, let us conclude this paper by showing the following corollary of Theorem 1.1 and Theorem 3.8 when $w=v \in A_{p}\left(\mathbb{R}^{n} \times \mathbb{R}^{m}\right)$.

Corollary 3.12 Let $\sigma, N \geq 1$ and $\mathcal{D}_{1}, \mathcal{D}_{2} \in \mathcal{F}(\sigma, N)$, and let $f$ be a weakly differentiable functions on $\mathcal{D}=\mathcal{D}_{1} \times \mathcal{D}_{2} \subset \mathbb{R}^{n} \times \mathbb{R}^{m}$. Let $W_{1}$ (W $\left.W_{2}\right)$ be a collection of cubes in $\mathcal{D}_{1}\left(\mathcal{D}_{2}\right)$ that satisfies the chain condition. If $w \in A_{p}\left(\mathbb{R}^{n} \times \mathbb{R}^{m}\right)$, $1<p<\infty$, then

$$
\| f-\left.f_{I_{0} \times J_{0}}\right|_{L_{w}^{p}(\mathcal{D})} \leq\left. C\left|I_{0}\right|^{1 / n}|| \nabla_{1} f\right|_{L_{w}^{p}(\mathcal{D})}+\left.C\left|J_{0}\right|^{1 / n}|| \nabla_{2} f\right|_{L_{w}^{p}(\mathcal{D})}
$$

where $I_{0}$ and $J_{0}$ are the 'central cubes' in $W_{1}$ and $W_{2}$ respectively.

Proof. First note that it follows from Theorem 1.1 that for each parallelepiped $R=I \times J$ in $\mathbb{R}^{n} \times \mathbb{R}^{m}$, we have

$$
\left\|f-f_{I \times J}||_{L_{w}^{p}(I \times J)} \leq C|I|^{1 / n}|| \nabla_{1} f\right\|_{L_{w}^{p}(I \times J)}+\left.C|J|^{1 / n}|| \nabla_{2} f\right|_{L_{w}^{p}(I \times J)} .
$$

Thus by similar argument as in the proof of Theorem 3.9, the result follows.

\section{References}

[1] R. C. Brown and D. B. Hinton, Weighted interpolation inequalities of sum and product form in $\mathbb{R}$, Proc. London Math. Soc., 56, no. 3 (1988), 261-280.

[2] R. C. Brown and D. B. Hinton, Weighted interpolation inequalities and embeddings in $\mathbb{R}$, Canad. J. Math., 62, no. 6 (1990), 959-980.

[3] C. Carton-Lebrun and H. P. Heinig, Weighted norm inequalities involving gradients, Proc. Roy. Soc. Edinburgh Sect. A, 112, no. 3-4 (1989), $331-341$.

[4] S. Chanillo and R. L. Wheeden, Poincaré inequalities for a class of non$A_{p}$ weights, Indiana Math. J., 41, no. 3 (1992), 605-623.

[5] Seng-Kee Chua, Extension theorems on weighted Sobolev spaces, Indiana Math. J., 41, no. 4 (1992), 1027-1076.

[6] Seng-Kee Chua, Weighted Sobolev's inequalities on domains satisfying the chain condition, Proc. Amer. Math. Soc., 117, no. 2 (1993), 449-457. 
[7] Seng-Kee Chua, Weighted Sobolev's inequalities on certain domains, Journal of London Math. Soc. (2), 51 (1995), 532-544.

[8] D. E. Edmunds, A. Kufner and Jiong Sun, Extension of functions in weighted Sobolev spaces, Rend. Accad. Naz. Sci. XL Mem. Mat. (5), $\mathbf{1 0 8}^{0}$, vol. 16, fasc. 17 (1990), 327-339.

[9] R. Fefferman and E. M. Stein, Singular integrals on product spaces, Adv. Math., 45 (1982), 117-143.

[10] R. Fefferman, Multiparameter Fourier Analysis, Beijing Lectures in Harmonic Analysis, Princeton University Press, 1986, 47-130.

[11] C. E. Gutierrez and R. L. Wheeden, Sobolev interpolation inequalities with weights, Trans. Amer. Math. Soc., 323 (1991), 263-281.

[12] T. Iwaniec and C. A. Nolder, Hardy-Littlewood inequality for quasiregular mappings in certain domains in $\mathbb{R}$, Ann. Acad. Sci. Fenn. Series A. I. Math., 10 (1985), 267-282.

[13] P. Jones, Quasiconformal mappings and extendability of functions in Sobolev spaces, Acta Math., 147, no. 1-2 (1981), 71-88.

[14] A. Kufner, Weighted Sobolev Spaces, John Wiley \& Sons Ltd, 1985.

[15] C. Pérez, Two weighted norm inequalities for Riesz Potentials and uniform $L^{p}$-weighted Sobolev inequalities, Indiana Math. J., 39, no. 1 (1990), $31-44$.

[16] E. Sawyer and R. L. Wheeden, Weighted inequalities for fractional integrals on Euclidean and homogeneous spaces, Amer. J. Math., 114, no. 4 (1992), 813-874.

[17] Xianliang Shi and A. Torchinsky, Poincaré and Sobolev inequalities in product spaces, Proc. Amer. Math. Soc., 118, no. 4 (1993), 1117-1124.

[18] Jan-Olov Stromberg and A. Torchinsky, Weighted Hardy Spaces, Lecture Notes in Mathematics, No. 1381, Springer, New York, 1989.

[19] Jan-Olov Stromberg and R. Wheeden, Fractional integrals on weighted $H^{p}$ and $L^{p}$ spaces, Trans. Amer. Math. Soc., 287 (1985), 293-321. 